\title{
Comprehensive intra/extracellular myocardial structural and functional characterization of hypertensive heart disease phenotypes
}

\author{
Jonathan C Rodrigues ${ }^{1 *}$, Antonio Matteo Amadu 1,2, Amardeep Ghosh Dastidar', Gergely V Szantho1, \\ Cattleya Godsave ${ }^{1}$, Laura E Ratcliffe ${ }^{3}$, Amy E Burchell ${ }^{3}$, Emma C Hart ${ }^{3}$, Mark Hamilton'1 , Angus K Nightingale ${ }^{3}$, \\ Julian F Paton ${ }^{3}$, Nathan E Manghat ${ }^{1}$, Chiara Bucciarelli-Ducci ${ }^{1}$
}

From 19th Annual SCMR Scientific Sessions

Los Angeles, CA, USA. 27-30 January 2016

\section{Background}

The European Society of Cardiology recognizes different hypertensive heart disease (HHD) phenotypes. HHD can be classified into 4 left ventricular (LV) phenotypes by indexed LV mass, mass to volume ratio $(\mathrm{M}: \mathrm{V})$ and indexed end diastolic volume (EDV) (Table 1). All remodeling/ hypertrophy phenotypes carry adverse cardiovascular prognosis, but the underlying mechanisms are incompletely understood. We investigated differences in intra/extracellular myocardial structure and function between phenotypes with T1 mapping and myocardial strain analysis.

\section{Methods}

88 treated hypertensive patients ( $49 \pm 14$ years, $57 \%$ male, SBP: $167 \pm 30 \mathrm{mmHg}$, DBP: $96 \pm 14 \mathrm{mmHg}$ ) underwent 1.5T CMR and were compared with 29 age- and sexmatched normotensive controls (47 \pm 14 years, $59 \%$ male, SBP: $128 \pm 12 \mathrm{mmHg}$, DBP: $79 \pm 10 \mathrm{mmHg}$ ).

Native and post-contrast T1 myocardial values were measured with a modified look-locker inversion-recovery sequence. Extracellular volume (ECV) and myocardial cell volume fractions were calculated. Circumferential strain values were estimated by voxel-tracking.

\section{Results}

There was a gradient of increasing LV mass from normal, to remodeling, concentric LVH and eccentric LVH $\left(70 \pm 9 \mathrm{~g} / \mathrm{m}^{2}\right.$ vs $75 \pm 10 \mathrm{~g} / \mathrm{m}^{2}$ vs $108 \pm 24 \mathrm{~g} / \mathrm{m}^{2}$ vs 122

${ }^{1}$ CMR Unit, NIHR Cardiovascular Biomedical Research Unit, Bristol Heart Institute, Bristol, United Kingdom

Full list of author information is available at the end of the article
Table 1 CMR definitions of patterns of hypertensive heart disease

\begin{tabular}{lccc}
\hline & $\begin{array}{c}\text { Indexed LV mass (g/ } \\
\text { m2) }\end{array}$ & $\begin{array}{c}\text { Indexed EDV (ml/ } \\
\text { m2) }\end{array}$ & $\begin{array}{c}\text { M/V (g/ } \\
\text { ml) }\end{array}$ \\
\hline $\begin{array}{c}\text { Normal LV } \\
\text { LV }\end{array}$ & $\leftrightarrow$ & $\leftrightarrow$ & $\leftrightarrow$ \\
$\begin{array}{c}\text { remodeling } \\
\text { Concentric } \\
\text { LVH }\end{array}$ & $\leftrightarrow$ & $\downarrow$ & $\uparrow$ \\
Eccentric LVH & $\uparrow$ & $\leftrightarrow$ & $\uparrow$ \\
\hline
\end{tabular}

$\mathrm{LV}$ mass = left ventricular mass, EDV = end-diastolic volume, $\mathrm{M} / \mathrm{V}=$ mass : volume ratio, $\mathrm{LVH}=$ left ventricular hypertrophy. LVM and EDV are indexed to body-surface area

$\pm 30 \mathrm{~g} / \mathrm{m}^{2}, \mathrm{p}<0.05$ respectively). This was due to stepwise increases in both: 1) indexed myocardial cell volume (eccentric LVH: $82 \pm 20 \mathrm{ml} / \mathrm{m}^{2}$ vs concentric LVH: $77 \pm 16 \mathrm{ml} / \mathrm{m}^{2}$ vs remodeling: $57 \pm 9 \mathrm{ml} / \mathrm{m}^{2}$ vs normal: $51 \pm 7 \mathrm{ml} / \mathrm{m}^{2}, \mathrm{p}<0.05$ respectively) and indexed interstitial volume (eccentric LVH: $33 \pm 10 \mathrm{ml} /$ $\mathrm{m}^{2}$ vs concentric LVH: $30 \pm 10 \mathrm{ml} / \mathrm{m}^{2}$ vs remodeling: $19 \pm 2 \mathrm{ml} / \mathrm{m}^{2}$ vs normal: $18 \pm 3 \mathrm{ml} / \mathrm{m}^{2}, \mathrm{p}<0.05$ respectively).

Eccentric LVH had significantly impaired peak circumferential strain $(-13 \pm 5 \%$ vs concentric LVH: $-16 \pm 3 \%$ vs remodeling: $-17 \pm 3 \%$ vs normal: $-18 \pm 3 \%$ vs controls: -17 $\pm 3 \%, \mathrm{p}<0.05$ respectively), with evidence of both peak systolic $(-70 \pm 20 \% / \mathrm{sec}$ vs concentric LVH: $-98 \pm 20 \% / \mathrm{sec}$ vs remodeling: $-115 \pm 38 \% / \mathrm{sec}$ vs normal: $-107 \pm 28 \% / \mathrm{sec}$ vs controls: $-101 \pm 13 \% / \mathrm{sec}, \mathrm{p}<0.05$ respectively) and peak diastolic strain rate impairment $(65 \pm 21 \% / \mathrm{sec}$ vs concentric LVH: $82 \pm 23 \%$ /sec vs remodeling: $90 \pm 24 \%$ / sec vs normal: $102 \pm 26 \% /$ sec vs controls: $101 \pm 26 \% / \mathrm{sec}$, $\mathrm{p}<0.05$ respectively). 


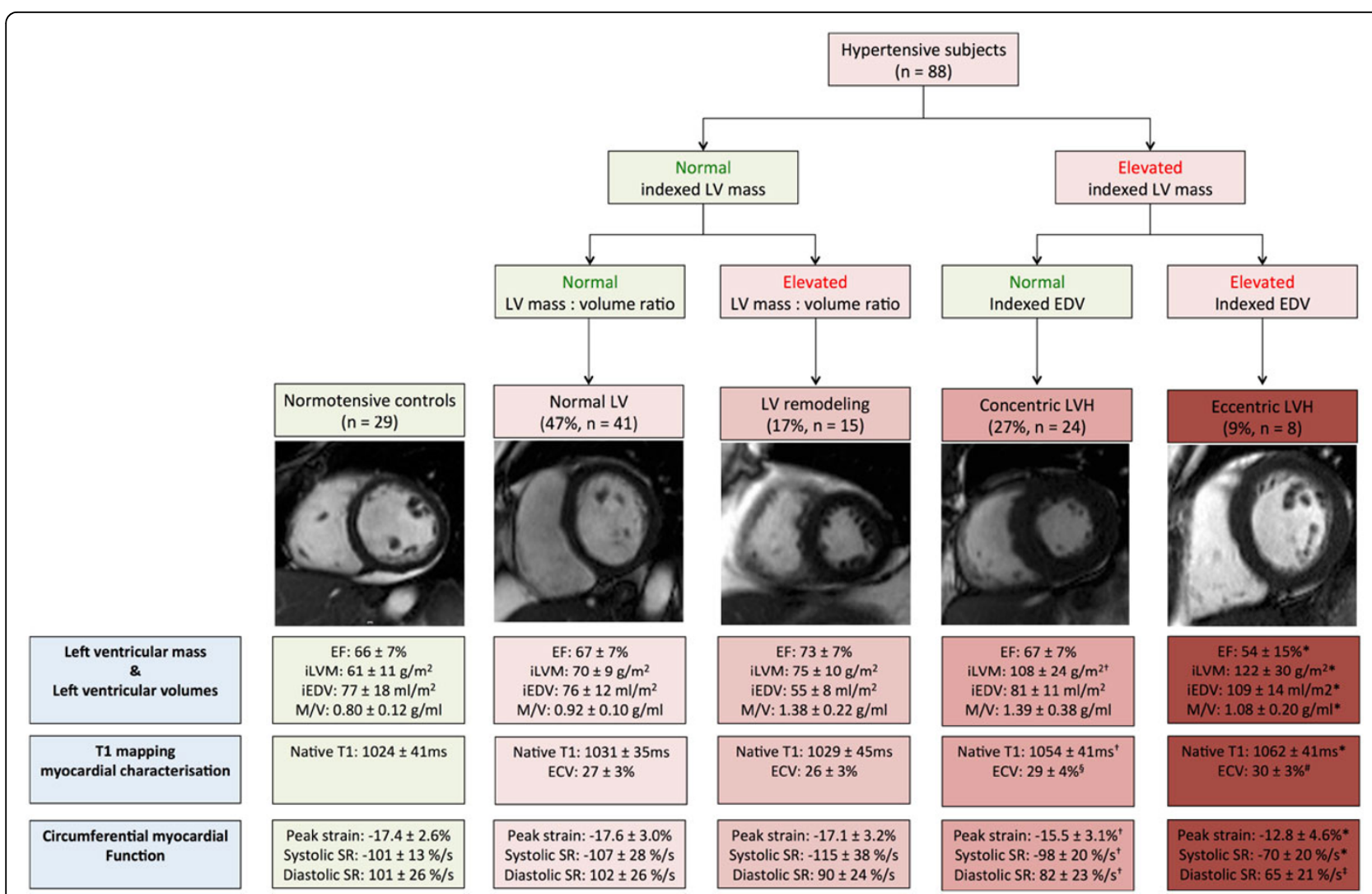

Figure $\mathbf{1} * \mathbf{P}<\mathbf{0 . 0 5}$, Eccentric LVH vs all subgroups. $+\mathrm{P}<0.05$, Concentric $L V H$ vs $L V$ remodeling vs normal LV vs controls. $\S P<0.05$, Concentric LVH vs LV remodeling. \# $P<0.05$, Eccentric LVH vs LV remodeling. $\neq P<0.05$, Eccentric $L V H$ vs $L V$ remodeling vs normal $L V$ vs controls

Despite similar BP severity as LVH, LV remodeling was associated with neither significant intracellular/interstitial expansion (native T1 $1029 \pm 45 \mathrm{~ms}$ vs $1024 \pm 41 \mathrm{~ms}$, $\mathrm{P}=0.67$ ), nor myocardial dysfunction compared to normotensive controls.

\section{Conclusions}

We comprehensively characterize, at the intra/extracellular myocardial level, structural differences between hypertensive phenotypes that are associated with functional consequences. We show that:

1) $\mathrm{LVH}$, in particular eccentric $\mathrm{LVH}$, is associated with significantly elevated myocardial cell volume, interstitial volume as well as myocardial systolic and diastolic strain impairment.

2) LV remodeling is associated with both normal myocardial structure and function.

Our results may help explain why eccentric LVH has poor prognosis. Our findings may have implications for future anti-hypertensive treatments.

\section{Authors' details}

'CMR Unit, NIHR Cardiovascular Biomedical Research Unit, Bristol Heart Institute, Bristol, United Kingdom. ${ }^{2}$ Department of Radiology, University of Sassari, Sassari, Italy. ${ }^{3}$ Cardionomics Research Group, Bristol Heart Institute, Bristol, United Kingdom.

Published: 27 January 2016

doi:10.1186/1532-429X-18-S1-P228

Cite this article as: Rodrigues et al:: Comprehensive intra/extracellular myocardial structural and functional characterization of hypertensive heart disease phenotypes. Journal of Cardiovascular Magnetic Resonance 2016 18(Suppl 1):P228. 